\section{IMMUNOGENOMIC EVALUATION OF CLEAR CELL RENAL CARCINOMA UNCOVERS HK3 AS A MYELOID SPECIFIC METABOLIC ENZYME}

${ }^{1}$ Bradley Reinfeld*, ${ }^{1}$ Matthew Madden, ${ }^{1}$ Melissa Wolf, ${ }^{2}$ Agi de Cubas, ${ }^{2}$ Scott Haake, ${ }^{2}$ Rachel Hongo, ${ }^{1}$ Margaret Axelrod, ${ }^{2}$ Jackie Bader, ${ }^{3}$ Aleksandar Obradovic, ${ }^{1}$ Dalton Greenwood, ${ }^{2}$ Xiang Ye, ${ }^{4}$ Justin Balko, ${ }^{2}$ Katy Beckermann, ${ }^{5}$ Benjamin Vincent, ${ }^{2}$ Brian Rini, ${ }^{3}$ Charles Drake, ${ }^{2}$ Jeffrey Rathmell, ${ }^{2}$ W Rathmell. ${ }^{1}$ Vanderbilt University School of Medicine, Nashville, TN, USA; ${ }^{2}$ Vanderbilt University Medical Center, Nashville, TN, USA; ${ }^{3}$ Columbia University Medical Center, New York City, NY, USA; ${ }^{4}$ Vanderbilt University Medical Center, Nashville, TN, USA; ${ }^{5}$ University of North Carolina, Chapel Hill, NC, USA

Background Glucose fixation is a hallmark clear cell renal carcinoma (ccRCC). ${ }^{12}$ Our group has shown unique metabolic enzyme utilization between malignant cells and infiltrating cells. Additionally, we uncovered the glycolytic nature of tumor infiltrating myeloid cells. ${ }^{3}$ Therefore, we decided to investigate the role of the hexokinase isoforms (HK1,2/3, GCK, and HKDC1) in the ccRCC tumor microenvironment (TME).

Methods For this study, we performed immunogenomic analyses across ccRCC samples available via The Cancer Genome Atlas (TCGA). ${ }^{4} 5$ Additionally, we examined the expression of hexokinases in the neoadjuvant VEGF inhibitor setting ${ }^{6}$ as well as correlation to a poor prognostic macrophage subset. ${ }^{7}$ Our group also performed single cell-ATAC seq on methocult cultures to further characterize the metabolic features of hematopoiesis. We additionally implemented qPCR on magnetically sorted bone marrow as well as myeloid cell culture to further interrogate the role of HK3 in macrophage biology and insitu RNA hybridization (RNA-ISH) to describe the subpopulation of HK3 + cells in the ccRCC TME.

Results Gene set enrichment analysis confirmed HK1/2's role in anabolic metabolism. GCK was barely detectable in these samples while HKDC1 expression decreased in ccRCC tumors. Intriguingly, patients with elevated expression of HK3 had an enrichment of interferon gamma response signature. In our evaluation of the TCGA, only HK3 expression correlated with poor outcome in ccRCC. CiberSortX demonstrated that HK3 expressing tumors correlated with the presence M2 macrophages while other HK family enzymes had marginal association with immune infiltrate. HK3 was the only hexokinase found to be significantly elevated with neoadjuvant pazopanib treatment in addition to being enriched in ccRCC patients with high levels of poor prognostic macrophages. RNA-ISH confirms HK3 expression is limited to myeloid cells in ccRCC tumors. The myeloid specific nature of $\mathrm{HK} 3$ is supported by transcript analysis from MC38 tumors, and qPCR on mouse bone marrow. Myeloid specificity for HK3 isoform expression is not restricted to malignancy; HK3 is one of a handful of genes that define myeloid identity from scATAC sequencing of in vitro differentiated CD34+ hematopoietic stem cells. Our ongoing in vitro studies indicate that M1 polarization (+LPS/ IFNg) increases expression of $\mathrm{HK} 1 / 2 / 3$, consistent with the anabolic phenotype of activate macrophages. However, stimulation with IFNg alone only elevates the expression of HK3.

Conclusions HK3 is a myeloid specific interferon gamma responsive gene, whose expression imparts poor prognosis for ccRCC cancer patients, while HK1/HK2 contribute significantly to the glucose uptake/pseudohypoxic phenotype seen throughout the ccRCC TME.

Acknowledgements N/a

Trial Registration N/A

\section{REFERENCES}

1. Courtney KD, et al. Isotope tracing of human clear cell renal cell carcinomas demonstrates suppressed glucose oxidation in vivo. Cell metabolism 2018;28(5):793800.e2.

2. Linehan WM, et al. The metabolic basis of kidney cancer. Cancer Discov 2019;9 (8): 1006-1021.

3. Reinfeld $\mathrm{BI}$, et al. Cell-programmed nutrient partitioning in the tumour microenvironment. Nature 2021.

4. Ricketts $\mathrm{CJ}$, et al. The cancer genome atlas comprehensive molecular characterization of renal cell carcinoma. Cell reports 2018;23(1):313-326.e5.

5. Creighton $\mathrm{CJ}$, et al. Comprehensive molecular characterization of clear cell renal cell carcinoma. Nature 2013:499(7456):43-49.

6. Wood CG, et al. Neoadjuvant pazopanib and molecular analysis of tissue response in renal cell carcinoma. JCI Insight 2020;5(22).

7. Obradovic $A$, et al. Single-cell protein activity analysis identifies recurrence-associated renal tumor macrophages. Cell 2021;184(11):2988-3005.e16.

Ethics Approval This clinical trial [in Reference 6] was approved by the IRBs at the University of Carolina at Chapel Hill (Office of Human Research Ethics) and MD Anderson (Office of Human Subjects Protection), and the research was conducted according to the Declaration of Helsinki principles. All participants provided written informed consent before the initiation of any research procedures. The studies were conducted in accordance with the guidelines approved by the Institutional Review Board (IRB) protocols, AAAO5706 and AAAA9967, respectively. Patients provided consent prior to taking part in the study. This is the clinical data take from the study in reference 7.All other studies referenced in the above abstract were conducted in accordance with the Declaration of Helsinki principles under a protocol approved by the Vanderbilt University Medical Center (VUMC) Institutional Review Board (protocol no. 151549). Informed consent was received from all patients before inclusion in the study by the Cooperative Human Tissue Network at VUMC. This is the clinical data take from the study in reference 3 and 7All mouse procedures were performed under Institutional Animal Care and Use Committee (IACUC)-approved protocols from VUMC and conformed to all relevant regulatory standards. The mouse protocol ID is 19000125

http://dx.doi.org/10.1136/jitc-2021-SITC2021.906 